\title{
Assistência de enfermagem ao paciente em hemodiálise: investigação completiva
}

\author{
Nursing care for hemodialysis patients: complementary research \\ Atención de enfermería al paciente en hemodiálisis: investigación complementaria
}

\section{Resumo}

O presente estudo tem como objetivo analisar aspectos que influenciam na vida do paciente hemodialítico, evidenciar o papel do enfermeiro frente à assistência prestada ao paciente em tratamento de hemodiálise e, por fim, elencar as complicações recorrentes aos pacientes submetidos à hemodiálise.Trata-se de um estudo de revisão integrativa da literatura, no qual os critérios de inclusão traçados foram artigos originais e nacionais com até cinco anos de publicação. Foram realizadas buscas de publicações nas seguintes bases de dados: Banco de Dados em Enfermagem, Literatura Latino-Americana e do Caribe em Ciências da Saúde e biblioteca eletrônica Scientific Electronic Library Online. Foram identificados 287 artigos entre as bases de dados utilizadas e selecionados 20 . O maior número de artigos foi encontrado no ano de 2016. A região com maior número de estudos utilizados foi a Centro Oeste com 50\% das publicações destacando os estudos nos estados de Mato Grosso e Distrito Federal. Evidencia-se que vários estados brasileiros sofrem com a doença renal crônica, tornando-se um problema de saúde pública no Brasil e no mundo. Entretanto, muitos indivíduos desconhecem a doença dificultando o diagnóstico, fazendo-se necessário um rastreamento cauteloso para pessoas que apresentam um ou vários fatores de risco para a DRC.

Palavras-chave: Cuidados de enfermagem; Unidades hospitalares de hemodiálise; Insuficiência renal crônica. 


\begin{abstract}
This study aims to analyze aspects that influence the life of hemodialysis patients, highlight the role of nurses in the care provided to patients undergoing hemodialysis treatment and, finally, list the recurrent complications in patients undergoing hemodialysis. The integrative literature review study, in which the inclusion criteria outlined were original and national articles with up to five years of publication. Publications were searched in the following databases: Database in Nursing, Latin American and Caribbean Literature in Health Sciences and Electronic Library Scientific Electronic Library Online. 287 articles were identified among the databases used and 20 selected. The largest number of articles was found in 2016. The region with the highest number of studies used was the Midwest with $50 \%$ of publications highlighting studies in the states of Mato Grosso and the Federal District. It is evident that several Brazilian states suffer from chronic kidney disease, making it a public health problem in Brazil and worldwide. However, many individuals are unaware of the disease, making diagnosis difficult, making careful screening necessary for people who have one or more risk factors for CKD.
\end{abstract}

Keywords: Nursing care; Hemodialysis hospital units; Chronic renal failure.

\title{
Resumen
}

Este estudio tiene como objetivo analizar aspectos que influyen en la vida de los pacientes en hemodiálisis, destacar el papel del enfermero en la atención que se brinda a los pacientes en tratamiento de hemodiálisis y, finalmente, enumerar las complicaciones recurrentes en los pacientes en hemodiálisis.. El estudio integrador de revisión de la literatura, en el que los criterios de inclusión planteados fueron artículos originales y nacionales con hasta cinco años de publicación. Las publicaciones se buscaron en las siguientes bases de datos: Base de datos en enfermería, Literatura latinoamericana y caribeña en ciencias de la salud y Biblioteca electrónica Biblioteca electrónica científica en línea. Se identificaron 287 artículos entre las bases de datos utilizadas y se seleccionaron 20. El mayor número de artículos se encontró en 2016. La región con mayor número de estudios utilizados fue el Medio Oeste con un 50\% de publicaciones destacando estudios en los estados de Mato Grosso y el Estado Federal. Distrito. Es evidente que varios estados brasileños padecen enfermedad renal crónica, lo que la convierte en un problema de salud pública en Brasil y en todo el mundo. Sin embargo, muchas personas desconocen la enfermedad, lo que dificulta el diagnóstico, lo que hace necesario un examen cuidadoso de las personas que tienen uno o más factores de riesgo de ERC.

Palabras clave: Atención de enfermería; Unidades de hemodiálisis hospitalarias; Insuficiencia renal crónica.

\section{Introdução}

As doenças crônicas (DC) demandam, interferências, relacionadas a modificação dos aspectos comportamentais permanente, em razão de integrar uma adversidade de enorme amplitude que corresponde cerca de $72 \%$ dos óbitos (Gesualdo et al., 2020).

A hemodiálise é o método mais empregado no Brasil para o tratamento da doença renal crônica (DRC), que é uma alteração heterogênia que afeta tanto a estrutura quanto a função renal, tendo a deficiência de calorias de diversos nutrientes essenciais (Sostisso et al., 2020).

O aumento da prevalência da DRC, e consequentemente a sua repercussão na morbimortalidade de pessoas portadoras dessa enfermidade, tornou-se um problema de saúde pública mundial. Uma importante complicação da DRC é o risco cardiovascular aumentado, considerado a probabilidade de um indivíduo vir a sofrer de uma doença cardiovascular, e que pode levar a frequentes internações e impactar a vida social e econômica do sujeito doente, da sua família e, ainda, da sociedade (Pinho, Silva \& Pierin, 2016).

As principais causas da DRC são: a Hipertensão Arterial Sistêmica, diabetes mellitus, doenças renais (glomerulopatia, nefropatia tubulointerticial, doença renal policística, displasia, hipoplasia renal) e uropatias (infecções urinárias de repetição, obstruções urinárias e cálculos urinários) (Pires et al., 2017).

Na América Latina, a incidência da DCR, no ano de 2004, era de 147 pacientes por milhão de população, variando de 11 por milhão de população na Guatalema e 337 em Porto Rico. Nos Estados Unidos, após elevação nas taxas de incidência dessa patologia, tem ocorrido uma estabilização com 363 por milhão de população. Outros países com elevadas taxas de incidência para esta condição de saúde são Taiwan sendo 418 por milhão de população e Japão com 275 por milhão de população (Louvison, 2016). Em julho de 2017, havia 133.464 pacientes em tratamento dialítico crônico, essas estimativas permanecem 
crescendo constantemente (Sesso et al., 2017).

As clínicas de serviços de nefrologia contam com a equipe de enfermagem para prestação da assistência, estes devem inspecionar o local de inserção do cateter, como também na fístula arteriovenosa, atentando-se aos sinais que indiquem o trauma vascular, afim de que seja mensurado a velocidade de infusão durante a hemodiálise (Spigolon et al., 2018).

Dentre os cuidados de enfermagem prioritários ao paciente durante o tratamento hemodialitico destacam-se a monitoração dos sinais vitais a cada trinta minutos, monitorar o peso do paciente antes e depois da diálise, examinar vias de acesso para hemodiálise e monitorar sinais flogísticos, adotar medidas para controle de infecções, proporcionar suporte emocional, avaliar dor e administrar analgésicos prescritos, e realizar massagens visando o relaxamento do paciente (Freitas \& Mendonça, 2016). A presença de uma equipe qualificada é de suma importância para evitar e identificar potenciais complicações (Nisio et al.,2017).

Diante disso, é importante que o enfermeiro esteja presente nas sessões de hemodiálise coordenando a equipe e identificando as necessidades particulares de cada paciente. Este profissional também deve intervir na educação da família e do paciente sobre a doença e suas complicações, fornecendo orientações sobre o plano terapêutico, com aspectos técnicos e psicológicos (Pires et al., 2017).

O estudo torna-se relevante diante da incidência nacional de pacientes renais crônicos em uso de hemodiálise. Sendo de fundamental importância a assistência de enfermagem junto ao paciente e à família, esclarecendo dúvidas e informações sobre os cuidados e sobre as implicações do tratamento na qualidade de vida do paciente. Objetiva-se analisar aspectos que influenciam na vida do paciente hemodialítico, evidenciar o papel do enfermeiro frente à assistência prestada ao paciente em tratamento de hemodiálise e, por fim, elencar as complicações recorrentes aos pacientes submetidos à hemodiálise.

\section{Metodologia}

O presente estudo utilizou como método, a revisão integrativa da literatura, que constitui uma ferramenta importante permitindo a análise de subsídios na literatura de forma ampla, além de divulgar dados científicos produzidos por outros autores. Destaca-se pela exigência dos mesmos padrões de rigor, clareza e replicação utilizados em estudos primários, além de compor a mais ampla abordagem metodológica referente às revisões de literatura (Cerqueira et al., 2018).

Para a elaboração da revisão as seguintes etapas foram percorridas: escolha do tema; estabelecimento de critérios para inclusão; busca na literatura; definição das informações relevantes a serem extraídas dos estudos selecionados; avaliação dos estudos incluídos seguida de uma composição resumida; interpretação dos resultados através de tabela; apresentação da discussão através do ponto de vista.

Dado o contexto formularam-se as seguintes categorias: aspectos que influenciam na vida do paciente hemodialítico, papel do enfermeiro frente ao paciente que realiza hemodiálise e complicações recorrentes aos pacientes submetidos à hemodiálise.

Utilizaram-se como critério de inclusão, artigos originais, na linguagem portuguesa, respeitando os limites de publicação entre 2016 a 2020, com acesso livre e disponível em texto completo online.

A coleta dos dados foi iniciada em junho a dezembro de 2020, sendo realizada por meio das bases de dados, LILACS (Literatura Latino-Americana e do Caribe em Ciências da Saúde), SciELO (Scientific Eletronic Library Online) e BDENF (Banco de Dados em Enfermagem). Através da utilização das terminologias em saúde: Cuidados de enfermagem; Unidades hospitalares de hemodiálise e insuficiência renal crônica, consultadas nos Descritores em Ciências da Saúde (DeCS), com o auxílio do operador booleano, que deram os seguintes cruzamentos: cuidados de enfermagem e unidades hospitalares de hemodiálise, unidades hospitalares de hemodiálise e DCR. 
Foi realizada uma leitura exploratória e seletiva, dos títulos e resumos dos artigos, para determinar as informações relacionadas ao tema e a partir dessa seleção, foram excluídas as pesquisas sem relevância ao tema do estudo. Posteriormente fez-se uma leitura analítica, com finalidade de agrupar as informações contidas nos artigos, a fim de responder os objetivos do estudo.

O instrumento de coleta de dados foi composto pelas variantes: autor e ano de publicação e resultados, por meio de quadro sintético. Na apresentação dos resultados, as informações de cada estudo, que se apresentaram com mais relevância à revisão, foram exibidas de modo descritivo. Organizados por meio de tabela, sucinta e individualmente para facilitar a análise crítica da amostra.

Por se tratar de uma pesquisa bibliográfica, os dados obtidos por meio dessa, seguiram princípios éticos propostos na lei dos direitos autorais 12.853/13, que dispõe em seu Art. $1^{\circ}$. Essa Lei regula os direitos autorais, entendendo-se sob esta denominação os direitos de autor e os que lhes são conexos.

\section{Resultados}

Foram identificados 287 artigos entre as bases de dados utilizadas e, selecionados 20. Desses, 11 foram extraídos da base de dados LILACS (Literatura Latino-Americana e do Caribe em Ciências da Saúde), 4 foram selecionados do Banco de Dados em Enfermagem - BDENF e os demais 5 artigos, da SciELO (Scientific Eletronic Library Online). Foram excluídos 267 artigos, sendo 98 artigos duplicados; 108 artigos de revisão e 66 artigos estavam fora do objetivo da pesquisa, uma vez que eles não obedeceram aos critérios de inclusão propostos na pesquisa, como está descrito no fluxograma abaixo. 
Fluxograma 1: Processo de seleção dos artigos indexados. Aracaju (SE), 2020.

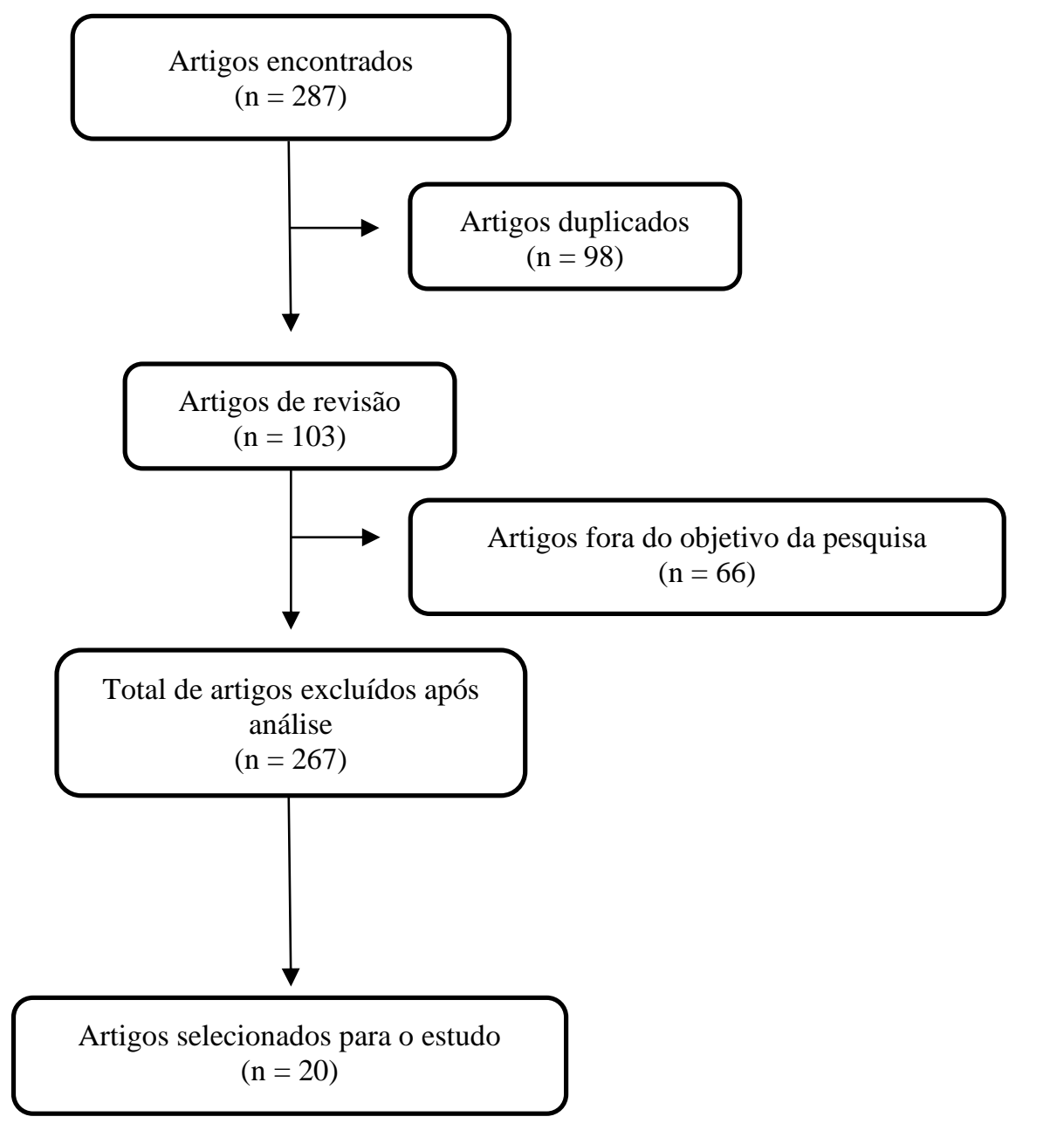

Fonte: Autores (2020).

A pesquisa contemplou publicações no idioma português, pré-estabelecido nos critérios de inclusão. Das bases de dados indexadas, a base online mais utilizada nesse trabalho foi a Literatura Latino-Americana e do Caribe em Ciências da Saúde (LILACS), como ilustrado no Gráfico 1.

Gráfico 1: Bases de dados mais utilizadas na revisão. Aracaju (SE), 2020.

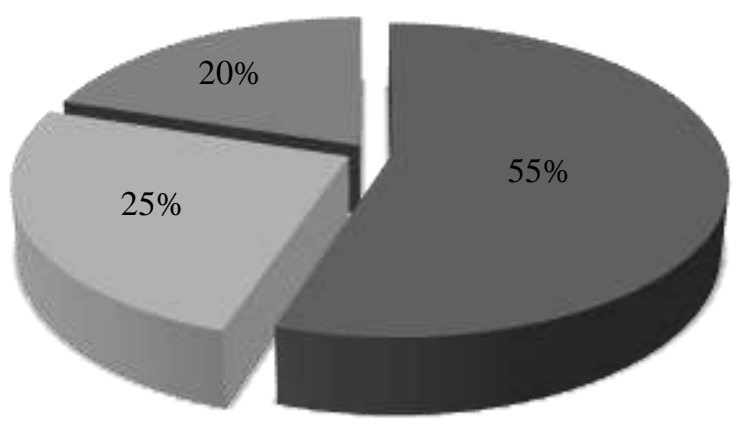

$$
\begin{aligned}
& \text { - LILACS } \\
& \text { SciELO } \\
& \text { BDENF }
\end{aligned}
$$

Fonte: Autores (2020). 
Frente aos resultados apresentados inicialmente teve-se um maior quantitativo de artigos encontrados na base de dados da Literatura Latino-Americana e do Caribe em Ciências da Saúde (LILACS), sendo assim uma das bases mais utilizadas pela maior amplitude de artigos nacionais e de melhor acesso e manuseio para filtragem dos critérios.

Constatou-se que o maior número de artigos foi encontrado no ano de 2016 sendo 10 desses, 05 em 2018, 03 em 2017 e 02 em 2019. Tais evidências foram avaliadas conforme o nível de importância acadêmica de cada temático nos respectivos anos. Conforme descrito no Gráfico 2.

Gráfico 2: Distribuição das publicações sobre a temática em questão, respeitando recorte temporal de 2016 a 2020. Aracaju (SE), 2020.

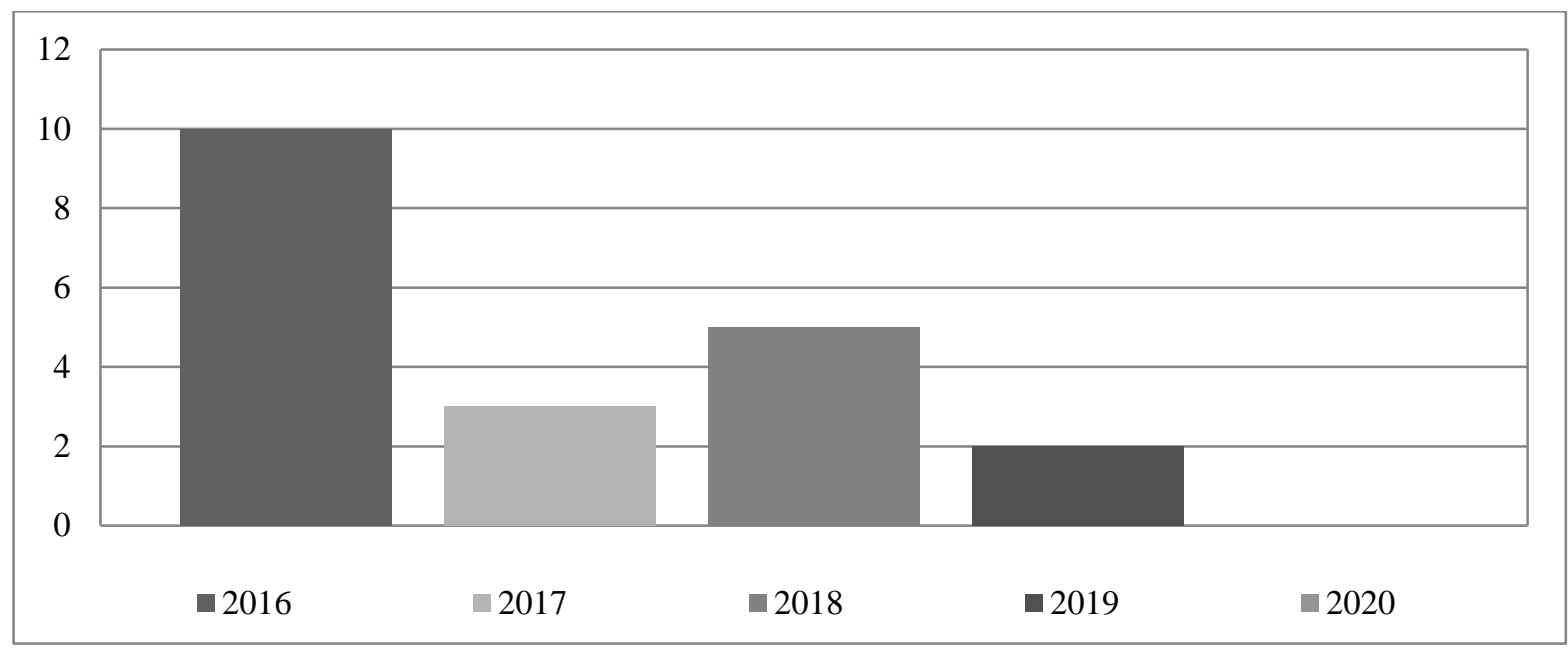

Fonte: Autores (2020).

Evidenciou a distribuição dos estudos utilizados na presente revisão por regiões do Brasil, onde a região com maior número de estudos utilizados foi a Centro Oeste com 50\% das publicações destacando os estudos nos estados de Mato Grosso do Sul e Distrito Federal. A região Sudeste foi responsável por 30\% dos estudos, sendo realizados nos estados do Rio de Janeiro e São Paulo, logo atrás a região Sul com 20\%, de estudos realizados no Rio Grande do Sul e Santa Catarina. Conforme descrito no Gráfico 3.

Gráfico 3: Distribuição dos estudos realizados nas respectivas regiões. Aracaju (SE), 2020.

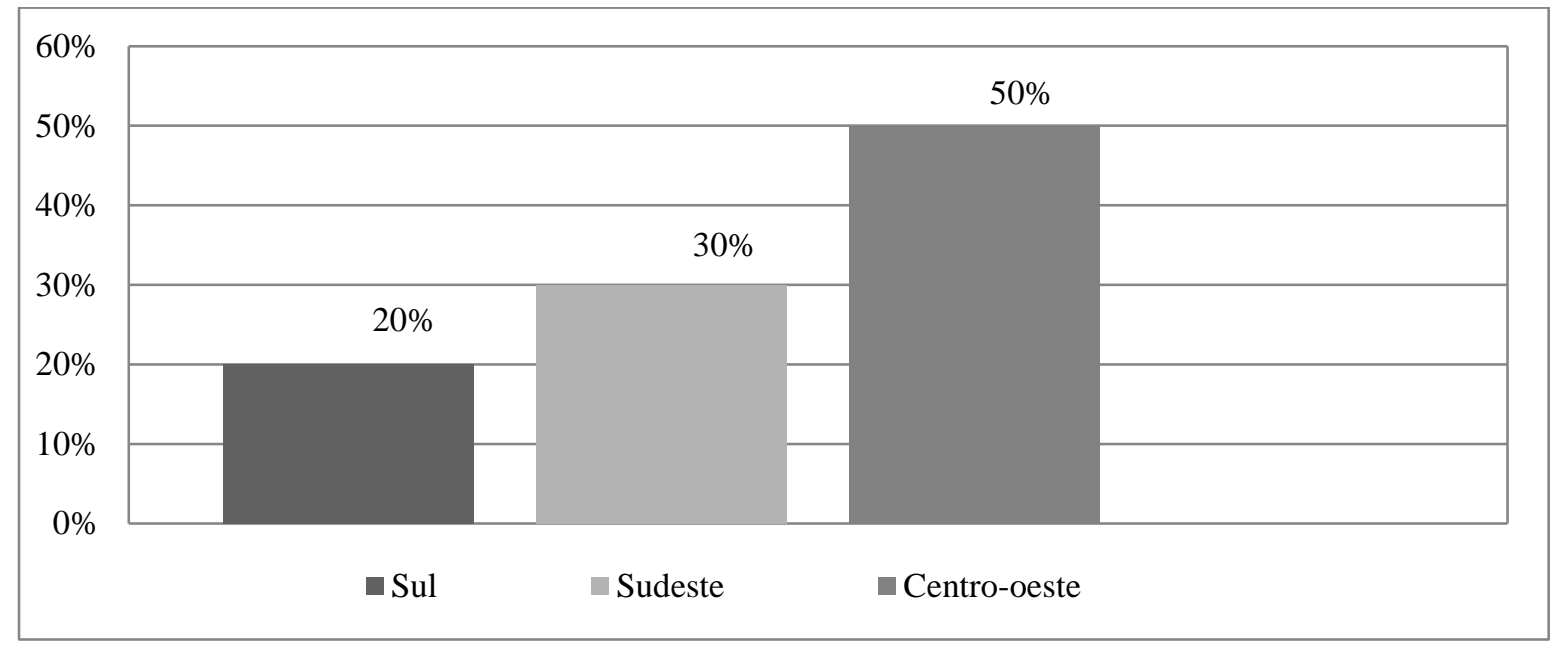

Fonte: Autores (2020). 
Considerando as pesquisas analisadas, evidencia-se que a maioria dos estudos (50\%) foram realizados em clínicas especializadas em hemodiálise com pacientes submetidos ao tratamento e profissionais atuantes na área nefrológica. Outra grande parte das pesquisas (45\%) foram realizadas em unidades hospitalares, e uma parcela mínima (5\%) realizada em unidades básicas de saúde, como descrito no Gráfico 4.

Gráfico 4: Distribuição das pesquisas realizadas nas respectivas unidades de atendimento ao paciente em tratamento hemodialítico. Aracaju (SE), 2020.

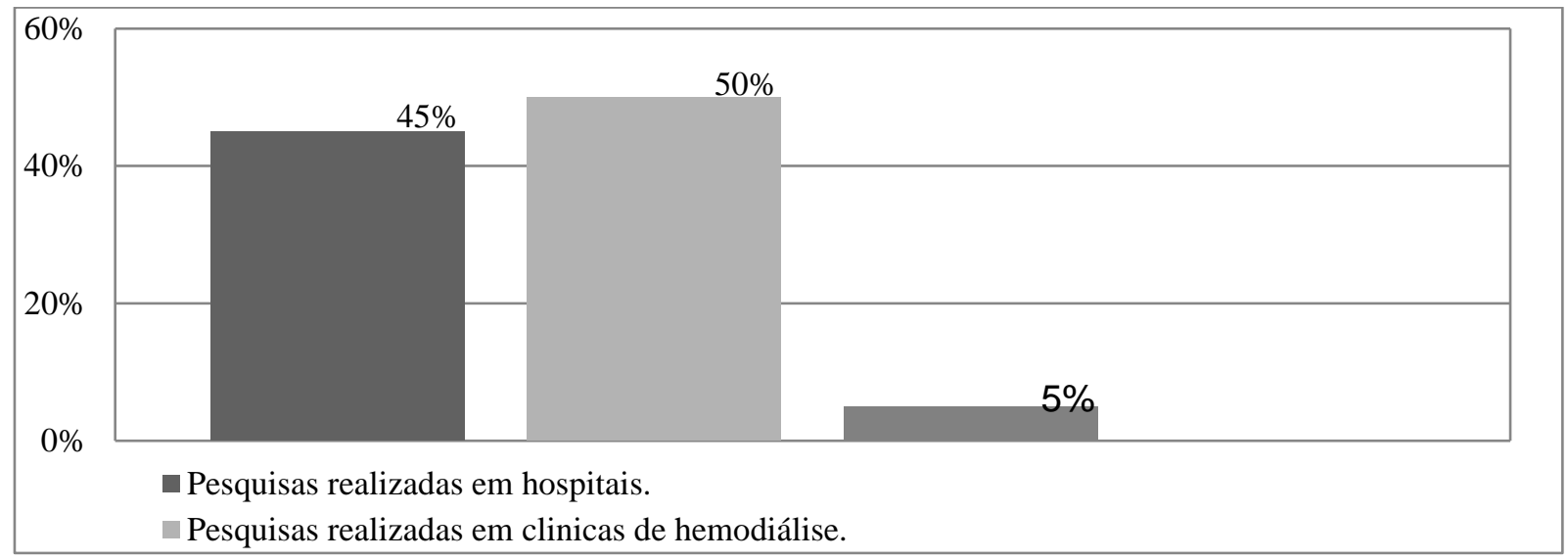

Fonte: Autores (2020).

Quadro 1: Síntese de artigos incluídos na revisão integrativa. Aracaju (SE), 2020. Complicações que podem influenciar na vida do paciente hemodialítico.

\begin{tabular}{|c|c|}
\hline AUTOR/ANO & $\begin{array}{l}\text { COMPLICAÇÕES RELATADAS PELOS PACIENTES E PROFISSIONAIS } \\
\text { ENVOLVIDOS: }\end{array}$ \\
\hline Pauletto et al., 2016a & $\begin{array}{l}\text { Estudo com pacientes em clínica hemodialítica conveniada ao SUS, os mesmos relataram } \\
\text { aspectos como: ansiedade, preocupação, medo iminente de morte, impotência sexual, } \\
\text { depressão e ideação suicida. }\end{array}$ \\
\hline Pinho, Silva \& Pierim, 2016 & $\begin{array}{l}\text { Estudo realizado em clínica médica com pacientes internados mostrou que } 30 \% \text { dos pacientes } \\
\text { apresentaram hipertensão arterial como complicação. }\end{array}$ \\
\hline Louvison et al., 2016 & $\begin{array}{l}\text { Já no estudo feito em centro de hemodiálise em São Paulo } 62 \% \text { dos pacientes estudados } \\
\text { apresentaram hipotensão com complicação, } 44 \% \text { apresentaram vômito e } 41 \% \text { vertigem. }\end{array}$ \\
\hline Marçal et al., 2019 & $\begin{array}{l}\text { Estudos realizados no Estado do Pará, com pacientes em tratamento hemodialítico. Anemia, } \\
\text { Hipertensão Arterial e Hiperfosfatemia, foram às complicações mais frequentes. }\end{array}$ \\
\hline Casas, Rodrigues \& Avila, 2016 & $\begin{array}{l}\text { Estudo realizado com uma população de pacientes renais crônicos em hemodiálise, os } \\
\text { mesmos elencaram complicações como: restrição alimentar, deformação dos ossos e anemia. }\end{array}$ \\
\hline Nisio et al., 2017 & $\begin{array}{l}\text { Estudo realizado em uma Clínica de Dialise, com pacientes, observou-se que as complicações } \\
\text { dominantes foram cardiovasculares e anemia, associada a perda de peso. }\end{array}$ \\
\hline Mattos \& Maruyama, 2019 & $\begin{array}{l}\text { Estudo foi realizado com usuários de hemodiálise, em uma clínica no Estado de Mato Grosso, } \\
\text { as complicações relatadas foram: febre, dor no corpo, fraqueza e até perda de movimentos nos } \\
\text { membros inferiores. }\end{array}$ \\
\hline Oliveira et al., 2016 & $\begin{array}{l}\text { Estudo realizado com pacientes da Santa Casa de Misericórdia de Itabuna, as complicações } \\
\text { mais frequentes desses pacientes foram: hipertensão, fraqueza e febre. }\end{array}$ \\
\hline
\end{tabular}


A importância do enfermeiro frente ao paciente em uso de hemodiálise

\begin{tabular}{|c|c|}
\hline AUTOR/ANO & RELATOS DO PAPEL DO ENFERMEIRO EM UNIDADES DE HEMODALISE \\
\hline Pires et al., 2017 & $\begin{array}{l}\text { Pesquisa realizada em uma clínica de hemodiálise, para os enfermeiros, os pontos de maior } \\
\text { relevância incluem: Orientar o paciente sobre o tratamento, número de sessões por semana, } \\
\text { conscientizá-los do tempo necessário para a filtração do sangue pela máquina, outros pontos } \\
\text { citados foram à avaliação do histórico do paciente, escolaridade existente, histórico familiar } \\
\text { para avaliar a pré-disposição genética, sinais vitais, exame físico e a rotina alimentar. }\end{array}$ \\
\hline Lemos et al., 2016 & $\begin{array}{l}\text { Estudo realizado em um Hospital das Clínicas da Região Metropolitana do Recife, com } \\
\text { enfermeiros atuantes na ala de hemodiálise, o estudo enfatiza a importância do enfermeiro } \\
\text { especialista em hemodiálise na manipulação de pacientes tão específicos como os em } \\
\text { tratamento hemodialítico. }\end{array}$ \\
\hline Spigolon et al., 2017 & $\begin{array}{l}\text { Estudo realizado em uma clínica especializado em nefrologia na região Noroeste do Paraná. O } \\
\text { enfermeiro e o profissional que assume o cuidado direto nesse tipo de tratamento, como a } \\
\text { identificação dos principais diagnósticos de enfermagem em indivíduos com doença renal } \\
\text { crônica em tratamento hemodialitico. }\end{array}$ \\
\hline Pauletto et al., $2016 \mathrm{~b}$ & $\begin{array}{l}\text { Salienta a importância do enfermeiro em manter os pacientes informados, quanto à } \\
\text { possibilidade de complicações durante as sessões e exposição de risco. }\end{array}$ \\
\hline Flores et al., 2018 & $\begin{array}{l}\text { Em um relato de experiência de acadêmicos de enfermagem, os mesmos ponderam evidenciar } \\
\text { a importância do enfermeiro frente aos pacientes em hemodiálise mediante a verificação de } \\
\text { sinais vitais, pesagem antes e a após a diálise, realização de exame físico, desenvolvimento da } \\
\text { sistematização da assistência de enfermagem e realização de curativos em fístula arteriovenosa } \\
\text { e cateter. }\end{array}$ \\
\hline Freitas \& Mendonça, 2016 & $\begin{array}{l}\text { O estudo mostrou que o enfermeiro tem o papel imprescindível no que se refere às intervenções } \\
\text { assistenciais do cuidado ao paciente, pois está à frente do planejamento e execução desses } \\
\text { cuidados. }\end{array}$ \\
\hline Oliveira, Silva \& Assad, 2016 & $\begin{array}{l}\text { Estudo realizado com profissionais de enfermagem em um hospital universitário no Rio de } \\
\text { Janeiro evidenciou-se a importância do enfermeiro nefrologista para realização dos cuidados } \\
\text { com as fístulas e cateteres no dia a dia e puncionar as fístulas. }\end{array}$ \\
\hline
\end{tabular}

Fonte: Autores (2020).

\section{Discussão}

No que diz respeito ao número de artigos publicados relacionado à temática de paciente renal crônico e em tratamento hemodialítico, é perceptível um elevado número de publicações no ano de 2016, o que representa a maior parcela de pesquisas indexadas na presente revisão, justificando-se pelos elevados índices de doentes renais crônicos e óbitos uma gama de publicações em decorrência dessas estatísticas (Nisio et al. 2017).

No ano de 2017 percebeu-se uma decrescente nas pesquisas, voltando-se a atenção acadêmica para o surto de dengue, zika e chikungunya, e consequentemente microcefalia. No ano de 2018 os assuntos voltados à insuficiência renal e seus tratamentos voltaram à ascendência, evidenciando as tecnologias do tratamento em hemodiálise e transplante renal, bem como maior acesso ao tratamento e sobrevida desses pacientes.

No período de 2019 a 2020, observou-se um novo declínio nas pesquisas relacionadas à temática, podendo justificar-se pela maior incidência de outras doenças virais, causando maior preocupação acadêmica para novas publicações, como exemplo o surgimento da pandemia do novo Coronavírus (COVID-19). 
Observou-se ainda que dentre os estudos encontrados, a região com o maior número de publicações foi a Centro Oeste, seguida pela Sudeste e por último a Sul. Isso se justifica pela maior facilidade de acesso ao serviço médico, assim como ao fato de que o incentivo financeiro para a realização de publicações cientifica também é mais elevado nessas regiões.

É importante mencionar que a DRC, por ser considerada uma doença progressiva, não apresentando sitomas em seu estágio inicial, é muito pouco identificada na atenção básica, o que comprova o baixissimo percentual de pesquisas realizadas nas UBS. Sob essa mesma ótica, o paciente homodialítico, na maiorias dos casos, só consegue detectar a DRC quando passa a ser sintomático, o que requer um tratamento mais avançado, que não pode ser ofertado pela UBS, mas sim nos hospitais e clínicas especializados.

\subsection{Categoria I: Aspectos que influenciam na vida do paciente hemodialítico}

O tratamento de hemodiálise ambulatorial tem duração média de quatro horas, três vezes por semana, conforme estado clínico do paciente. Essa terapêutica é acompanhada por diversas limitações e restrições, ocasionando mudanças significativas no cotidiano dos pacientes (Paulleto et al., 2016a).

Algumas restrições alimentares são importantes para o não agravamento do paciente nas sessões de hemodiálise e na vida cotidiana. As restrições de ingesta de líquidos requerem um esforço do paciente dialítico. Alimentos muito condimentados e doces também devem ser evitados por serem alimentos que causam sede.

As modificações que o tratamento na vida dos usuários, influenciam na vida emocional destes de maneira negativa. $\mathrm{O}$ conviver diário com a doença e o tratamento envolve repetição de ações, com presença de sofrimento contínuo e aflição (Alvez, Guedes \& Costa, 2016)

A hemodiálise requer cuidados complexos, pois é considerado um tratamento altamente invasivo, o qual demanda de uma assistência direta e contínua ao paciente tanto a nível domiciliar como pelos serviços que prestam esta terapia, podendo se sobrepor aos cuidados demandados pelo transplante (Oliveira et al., 2016).

Geralmente as dificuldades de adesão ao tratamento estão relacionadas a não aceitação da doença, à percepção de si próprio e ao relacionamento interpessoal com familiares e ao convívio social (Casas et al., 2016).

Nesse contexto, é importante que o enfermeiro ajude na integração do paciente com os familiares, uma vez que muitos pacientes chegam a suas residências com alguns sintomas, sentindo-se debilitado, e essa aproximação da família é capaz de fazer com que ela se coloque no lugar do paciente e, consequentemente, possam dar todo apoio necessário ao paciente dialítico.

Os sentimentos de desanimo e desespero podem estar associados às causas de abandono do tratamento, muitas vezes pela falta de orientação e suporte na adesão a terapia. Somando-se a isso, as limitações para a realização das atividades diárias, ansiedade, medo iminente de morte, impotência sexual, privações alimentares, depressão e ideação suicida (Marçal et al., 2019).

\subsection{Categoria II: Papel do enfermeiro frente ao paciente que realiza hemodiálise}

O cuidado e a manipulação dos cateteres e fístulas são considerados uma ação de grande importância realizada pelos profissionais de saúde que atuam, no procedimento de hemodiálise. Entende-se que é necessário que o profissional de saúde tenha especialidade em nefrologia, com conhecimento na prática que permite garantir segurança durante o momento da sua presença na sala de hemodiálise, e assim contribuir para uma assistência de forma humanizada nesses pacientes (Oliveira, Silva \& Assad, 2016).

Sendo assim o objetivo da assistência de enfermagem na área de nefrologia é identificar e monitorar os efeitos adversos da hemodiálise e complicações decorrentes da própria doença, desenvolvendo ações educativas de promoção, prevenção e tratamento. Bem como, realizar a educação e treinamento do paciente e familiar quanto aos novos hábitos de vida e cuidados com fístulas e dispositivos (Flores et al., 2018). 
O papel do enfermeiro deve focar na educação do paciente diante das cobranças determinadas pelo tratamento, pois o conhecimento mais profundo sobre sua doença, tratamento e possibilidades de reabilitação pode auxiliá-los no enfrentamento de situações que causam estresse vivenciado no cotidiano hemodialítico (Pires et al., 2017)

Sendo assim, nota-se que o enfermeiro no exercício do seu papel profissional, deve constituir ações educativas para propiciar um tratamento de qualidade e eficaz em benefício ao paciente, realizando um trabalho de prevenção e tratando as complicações. Deve ser ofertado um atendimento humanizado, integral, a fim de suprir as necessidades humanas básicas do indivíduo de modo que o satisfaça, criando uma relação de confiança e segurança entre paciente e o enfermeiro, dando prioridade aos cuidados necessários ao seu tratamento.

Contudo a importância da assistência de enfermagem para a inserção de uma equipe multiprofissional é fundamental para que esses pacientes entendam a necessidade da adesão ao tratamento e a manutenção de uma boa qualidade de vida.

\subsection{Categoria III: Complicações recorrentes aos pacientes submetidos à hemodiálise}

A hemodiálise gera complicações potenciais, entre elas: hipotensão e hipertensão arterial, câimbras musculares, síndrome do desequilíbrio da diálise, náuseas, vômitos, prurido, cefaleia, febres e calafrios, por isso o enfermeiro deve estar apto a intervir em tais intercorrências (Flores et al., 2018).

Complicações que envolvem a inserção do cateter são consideradas como tardias e imediatas - hemorragias, traumas vasculares, lesão de plexo braquial, embolia gasosa e hematomas são apenas algumas complicações imediatas. Quanto as tardias destacam-se: falência e obstrução do cateter, infecções no local da punção, estenose e oclusão da veia receptora do cateter (Oliveira et al., 2016)

A complicação mais comum é a hipotensão arterial, sendo ocasionadas por vários fatores como excesso de líquido removido, diferenças na temperatura e composição do dialisado, incompatibilidade da membrana do capilar com o sangue do paciente, dentre outras, que em consequência da queda na pressão arterial há presença de náuseas e vômitos, sudorese, tontura e taquicardia (Sancho, Tavarez \& Lago 2016).

As intercorrências que comumente ocorrem durante as sessões de hemodiálise são: as cãibras musculares, estando presentes em $20 \%$ das sessões de hemodiálise, acometendo principalmente os membros inferiores, devido a rápida saída de eletrólitos e líquido do meio extracelular, hiponatremia e hipotensão (Pires et al., 2017).

As complicações também podem estar presentes nos pacientes cardíacos, dentre elas destacam-se as arritmias cardíacas derivando de mudanças na quantidade de eletrólitos ou nos níveis de $\mathrm{pH}$, ou devido a retirada de antiarrítmicos durante a hemodiálise (Nisio et al., 2017). Já as complicações com menores índices de ocorrência em pacientes cardíacos, destacam-se a embolia pulmonar, ocasionando cefaleia, náuseas e intensa dor abdominal (Matos \& Maruyama, 2018).

Outra complicação gerada em pacientes em hemodiálise é a síndrome do desequilíbrio de diálise, que se origina com deslocamento do líquido cerebral, provocando no paciente crises de cefaleia, vômito, confusão mental, agitação, tremores, rebaixamento do nível de consciência e convulsões (Lemos et al., 2016)

Diante das possíveis complicações decorrentes da hemodiálise, nota-se a necessidade de ações rápidas com intervenções de enfermagem limitando a intercorrência e a intensidade das manifestações clínica no paciente durante o tratamento.

\section{Considerações Finais}

O estudo evidenciou que vários estados brasileiros sofrem com a DRC, tornando um problema de saúde pública no Brasil e no mundo. Muitos indivíduos desconhecem a doença dificultando o diagnóstico, sendo necessário um rastreamento cauteloso para pessoas que apresentam um ou vários fatores de risco para a DRC. 
Nota-se a necessidade da investigação e discursão, afim de que possa ser compreendido a implementação de técnicas nos procedimentos operacionais dos técnicos que atuam com o tratamento dos pacientes portadores DRC. No entanto

A atuação do profissional de saúde, em especial o enfermeiro, enquanto protagonista do atendimento a esse paciente, através de sua capacidade técnica, do diálogo e da atenção, facilita a adaptação do paciente a esse novo hábito de vida.

Ressalta-se que a área de nefrologia é amplo para os que atuam no campo da enfermagem, objetivando que os profissionais busquem entender as carências de seus pacientes, afim de que possam atender com mais veemência, traçando estratégias que busquem minimizar, os desconfortos provenientes do tratamento, assim como os psicológicos.

\section{Referências}

Alvez, L.O., Guedes, C.C.P. \& Costa, B.G. (2016). As ações do enfermeiro ao paciente renal crônico: reflexão da assistência no foco da integralidade. Revista de pesquisa cuidado é fundamental. 8(1), 3907-392.

Casas, J., et al. (2016). Educação nutricional para pacientes renais crônicos em programa de hemodiálise. Revista Nutrire. 40(1), 36-44.

Carvalho, P. F. (2020). Dissertação de Mestrado apresentada ao Programa de Pós-Graduação Ensino na Saúde da Universidade Federal de Ciências da Saúde de Porto Alegre, como requisito parcial para obtenção do título de Mestre em Ensino na Saúde.

Flores, A. D., et al. (2018). Ações em enfermagem na nefrologia: 24 anos de extensão. Revista expressa extensão. $22(2)$, 70-81.

Freitas, R. L. S. \& Mendonça, A. E. (2016). Cuidados de enfermagem ao paciente renal crônico em hemodiálise. Revista Cultural e Científica do UNIFACEX. $14(2), 22-35$.

Gesualdo, G. D., Duarte, J. G., Zazzetta, M. S., Kusumota, L. \& Orlandi. F. S (2020). Fragilidade e fatores de risco associados em pacientes com doença renal crônica em hemodiálise. Ciênc. saúde coletiva. 25(11).

Lemos, K. C. R. F., et al. (2016). Práticas científicas dos enfermeiros das clínicas de hemodiálise. Revista de enfermagem UFPI. 4(2), 69-75.

Louvison, M. C. P., et al. (2016). Prevalência de pacientes em terapia renal substitutiva no Estado de São Paulo. Boletim Epidemiológico Paulista. 8(95), 23-42.

Marçal, G. R., et al. (2019). Qualidade de vida de pessoas com doença renal crônica em hemodiálise. Revista cuidado é fundamental. 11(14), 908-913.

Matos, M. \& Maruyama, S. A. T. (2019) A experiência em família de uma pessoa com diabetes mellitus e em tratamento por hemodiálise. Revista eletrônica de enfermagem, 11(4), 971-981.

Nisio, J. N., et al. (2017). Impacto de um Programa de Educação Nutricional no Controle da Hiperfosfatemia de Pacientes em Hemodiálise. Revista Brasileira de nefrologia. 29(3), 153-157.

Oliveira, C. S., et al. (2016). Perfil dos pacientes renais crônicos em tratamento hemodialítico. Revista Baiana de enfermagem. 29(1), 42-49

Oliveira, N. B., Silva, F. V. C. \& Assad, L. G. (2016). Competências do enfermeiro especialista em nefrologia. Revista de enfermagem URJ. 23(3), 375-380.

Pauletto, M. R., et al. (2016. Percepção de pacientes para transplante renal sobre a hemodiálise fora da lista de espera. Revista de enfermagem da UFPE. 10(04), 1194-1201.

Pauletto, M. R., et al. (2016). Transplante renal: percepção de pacientes em hemodiálise fora da lista de espera. Revista de enfermagem da UFSM. 6(2), 154163.

Pinho, N. A., Silva, G. V. \& Pierin, A. M. G. (2017). Prevalência e fatores associados à doença renal crônica em pacientes internados em um hospital universitário na cidade de São Paulo, SP, Brasil. Jornal Brasileiro de Nefrologia. 37(1), 91-97.

Pires, M. G., et al. (2017). O papel da enfermagem na assistência ao paciente em tratamento hemodialítico. Revista Tendências da Enfermagem Profissional. $9(3), 2238-2244$.

Sancho, P. O. S., Tavarez, R. P. \& Lago, C. C. L. (2015). Assistência de enfermagem frente às principais complicações do tratamento hemodiálitico em pacientes renais crônicos. Revista de enfermagem contemporânea. 2(1), 183-169.

Sesso, R. C., Lopes, A. A., Thomé, F. S., Lugon, J. R. \& Martins, C. T. (2017). Inquérito Brasileiro de Diálise Crônica. J Bras Nefrol. 39(3), 261-6.

Spigolon, D. N., et al. (2018). Diagnósticos de enfermagem de portadores de doença renal em hemodiálise: estudo transversal. Revista Brasileira de Enfermagem, 71(4), 2130-2136.

Sostisso, C. F., Olikszechen, M., Sato, M. N., Oliveira, M. A. S. C. \& Karam, S. (2020). Força de preensão manual como instrumento de avaliação do risco de desnutrição e inflamação em pacientes em hemodiálise. Braz. J. Nephrol. (J. Bras. Nefrol.) 42(4),429-436. 